\title{
Review of Nature Inspired Algorithms
}

\author{
Sakshi Sukale \\ Mumbai University \\ D. J. Sanghvi College of Engineering, \\ Vile Parle, Mumbai, 400056, India
}

\author{
Tanaji D. Biradar \\ Mumbai University \\ D. J. Sanghvi College of Engineering, \\ Vile Parle, Mumbai, 400056, India
}

\begin{abstract}
In the recent years, lots of researches have researched many algorithms so as to improve performance of the wireless sensor network for to save energy as well as network life time. A mobile ad hoc network MANET is a collection of mobile users node that communicate over wireless links. Research interest in MANETs has been growing very fast, and particularly MANET routing protocols. Routing in MANET is become challenging because of constantly changing network topology, limited bandwidth and energy issues. Researchers have invented many algorithms by taking inspiration from the nature. The behavior of these algorithms is based on nature's self-organizing systems such as bird flocking, ant colonies and fish schools. In this paper we over viewed some nature inspired routing algorithms for mobile Ad-Hoc networks and compares them.
\end{abstract}

\section{General Terms}

Algorithms.

\section{Keywords}

MANET, Ad-Hoc, Routing.

\section{INTRODUCTION}

"Mobile Ad Hoc Network." is a type of ad hoc network that can change locations and configure itself on the fly. Because MANETS are mobile uses wireless connections for connecting different networks. These networks can be a communication between ground station to a satellite stations, WI-FI or any another medium like cellular network.

A mobile ad hoc network (MANET) is generally defined as a network that has many free or autonomous nodes, often composed of mobile devices or other mobile pieces that can arrange themselves in various ways and operate without strict top-down network administration.

Some MANETs are restricted to a local area of wireless devices (such as LAN for laptop, computer), while others may be connected to the Internet. For example, A VANET (Vehicular Ad Hoc Network) is a type of MANET which is communicating with roadside equipment. While most of the time vehicles do not have Internet connection. In this situation the roadside equipment which is already having Internet allows transmission of data from vehicles over the Internet. The vehicle data may be used to track traffic or trucking fleets. MANETs are not secure because of dynamic in nature, so it is important to be take care about which data is going to send over a MANET.

A mobile ad hoc network (MANET) is a non-centralized, multi hop, wireless network that lacks a common infrastructure and hence it needs self - organization. The biggest challenge in MANETs is to find a path between communicating nodes. The MANET routing problem occurs because the topology of the network changes constantly and paths which were initially efficient can quickly become inefficient or even infeasible as the bandwidth of the wireless network is limited and also information flow control in the network is restricted. Protocol is a standard that controls how mobile nodes decide which way to route packet between computing device to a mobile Ad-Hoc network. Different types of protocols are using for sending packet from Source to destination. Some basic types are listed below [1].

1) Proactive (Table Driven) routing protocols: this type of protocol maintains fresh list of destinations and their routes by periodically distributing routing tables throughout the network.

2) Reactive (On-Demand) routing protocol: this type of protocol finds a route on demand by flooding the network with route request packet

3) Hybrid (Reactive \& Proactive) Routing Protocol: this type of protocol combines the advantages of both type routing.

4) Hierarchical routing protocol: in this type the choice of proactive or reactive routing depends on the hierarchical level where a mobile node resides.

5) Adaptive (Situation aware) routing protocol: in this type the routing is initially established with some proactive prospected routes and then serve the demand from additionally activated routes through reactive flooding.

6) Flow Oriented routing protocol: this type of protocol find a route on demand by following present flows.

In this paper we are reviewing some important natured inspired algorithms. Swarm intelligence (SI) is the collective behavior of decentralized, self-organized systems, natural or artificial. The employment on artificial intelligence work is introduced by Gerardo Beni and Jing Wang in 1989, in cellular robotic systems.

SI systems are nothing but interaction between population of simple agents or boids with each other also with environment. The inspiration for such kind of system initially comes from nature like biological systems. These agents follow simple rules. However there is no centralized control for individual agent behavior, the local, and up to a certain degree random, interactions between these agents lead to the emergence of "intelligent" global behavior. Every individual agent should not have global information. In short we are reviewing algorithm like bird flocking, different types of ant colony algorithm and some extents to its merits and demerits.

\section{ROUTING ALGORITHM BASED ON BIRD FLOCKING}

These type of algorithms based on flocking behavior of birds Bird travels a long distance in flocks forming ' $\mathrm{V}$ ' shape. While traveling head of flock has to carry all burdens. But this down wash sphere head getting positive effect of up wash by rotating line vortices from opposite side. Resulting reduces the up thrust required by the other bird. Because of this a bird can travel a long distance in minimum energy. When the energy of head is reduces, it will replace by the other bird, 
using this way leader of the folk keep changing for energy conservation.

a) T.Srinivasan et.al. (2006)[2] Has explained Bird Flocking Behavior based Routing algorithm .The observation of this paper is that the nodes are sharing their information with neighbor like velocity and direction sharing in the birds folk. in this paper on -demand and hybrid routing protocol is proposed. These protocols are flood based route discovery. The RREQ (Route Request) packet is flooded across the network. Since the source node is not known the search is omni- directional. The Encounter Search algorithm is proposed to improve omni-directional search. This algorithm collects history of last communication between the nodes. The source node searches the most recent node by this history table. Depending on table the most recent node will be select for communication and this procedure repeats until it reaches to the destination. After route discovery the maintenance is perform by using Direction Forward Routing.

b) Mohit Tiwari et.al. [2010][3] Proposed Bird Flight Inspired Clustering based Routing Protocol for Mobile Ad Hoc Networks. In this paper the algorithm mainly maintaining the cluster. This is a position based routing algorithm and Dynamic Selection of gateway nodes so as to reduce control packet flooded in the network. These algorithms are very few insensitive to scaling of design variables hence it is simple to implement. Also these are derivative free, greater diversity, exploration over a single population. These algorithm having demerits is fast and premature convergence in mid optimum points and weak local search ability.

\section{ANT COLONY BASED ROUTING ALGORITHMS}

In these algorithms the basic idea is taken from food searching behavior of real ants. When ants are searching for food their route starts from their nest to the food. When an ant comes across intersection in the path, it has to decide which branch to take next. While walking, an ant deposits a pheromone on the same route. This is a volatile chemical substance which ants are able to smell and identify the route taken by the forwarded ant. The concentration of pheromone on certain path is an indication of its frequent usage. After a short time the path having high concentration will be the shorter path. A few algorithms we have explained further based on ant behavior.

a) Di Caro, Ducatelle, and Gambardella et.al. (2004) [4, 5, 6, 7, 8, 9, and 11], AntHocNet : In these paper they have explained routing algorithm is based on the nature-inspired ant colony optimization framework. It is a hybrid algorithm; it is reactive in the sense that a node only starts gathering routing information for a specific destination when a local traffic session needs to communicate with the destination and no routing information is available. It is proactive because the nodes pro-actively keep the routing information during the communication. This information is related to the ongoing flow also up-to-date with network changes for traffic and topology. The algorithm tries to find paths for minimum number of hops, good signal quality, and low congestion between adjacent nodes. The paths are decided by using Monte Carlo sampling method for agents like ant which are communicating in a stigmergic way. Nodes in Ant-Hoc Net algorithm forward data stochastically.

When a node has to take multiple hops to reach destination, the next hop for the node is depend on probability. This algorithm is more scalable than AODV but slow routing details spreading via hello message. Also overhead is more due to routing behavior of the reactive ants.

b) Guenes et al. (2002) [12] Have explained Ant-ColonyBased Routing Algorithm (ARA) in this paper .ARA is a strictly reactive algorithm. It does not use any HELLO packets to find its neighbors. HELLO packets are sent by the routers and this is used for calculate time delay for datagrams communication between its neighbors, these packets consist clock and time stamp information. When a packet arrives at a node, the node checks for routing information is available for destination ' $d$ ' in its routing table. Route discovery is done by two ways, one is by FANT flood technique and another is by FANT forward technique. In the FANT flooding technique, when a FANT arrives to any intermediate node, the FANT is flooded to all its neighbors. Node will check for routing table for destination information. When table found, it forwards the packet over that node or it broadcasts a FANT to find destination node. Also it will store a maximum hop count on the FANT. In the FANT forwarding scheme, when a FANT reaches an intermediate node, the node checks its routing table to find whether it has a route to the destination over of its neighbors. If neighbor is found, then FANT is forwarded to that neighbor or it will flood to all other neighbors as in the flood scheme. This algorithms node power is saved due sleep mode properties. This is highly adaptive, efficient and scalable, not generating any loop due to which overhead is very small as compared to other algorithms. c) Marwaha et al. (2002) [14] explained ACO Based on Demand Distance Vector (Ant- AODV). This is a hybrid protocol that is able to provide reduced end-to-end delay and high connectivity than AODV. AODV perform the reactive part and an ant-based approach performs the proactive one. The main target for the ant algorithm is to create routes continuously to reduce delay and the network latency, also increasing the probability of detecting routes faster. Ant- AODV's artificial pheromone model is based on to discover the network topology and number of hops, Route establishment in conventional antbased routing techniques is dependent on the ants visiting the node and providing it with routes. The nodes also have capability of launching on-demand route discovery to find routes to destinations. To increase the node connectivity the ants with AODV uses. It reduces the amount of route discoveries and also the delay time in route discovery. This makes the Ant-AODV hybrid routing protocol suitable for real-time data and multimedia communications. Ant- AODV uses route error (RERR) messages to inform upstream nodes of a local link failure. The routing table in Ant-AODV is common to both the ants and AODV. Frequent HELLO broadcasts are used to maintain a neighbor table. This is having less connection setup delay. The demerits of this algorithm are more bandwidth consumption and heavy overhead due to single request.

d) Baras and Mehta (2003) [15] have explained in this paper a Probabilistic Emergent Routing Algorithm (PERA) algorithm. In the paper they have discovered two algorithms. The first algorithm is a proactive which is similar to AntNet. Nodes maintain pheromone entries for all destinations by periodically launching forward ants, which take random selection for unbiased exploration, and information packets are deterministically routed over the paths with the best quality. The massive routing overhead and the inefficient route discovery of this algorithm led to PERA, and is purely reactive algorithm not very different from AODV. The forward ants are now flooded towards their destinations. This strategy can find out multiple paths. However, data packets are routed over the single best path available. Presence of 
multiple paths is helpful for quick recovery in case of link failures. The performance of the algorithm is as good as AODV on the basis of simulation experiments. In case of present link failure this can set another link hence advantage of strong path can be achieved by using this algorithm. One disadvantage of overhead is there due to multiple path setting.

e) Heissenb Uttel and Braun (2003) [18] have explained Mobile Ant-Based Routing (MABR). This is based on geographical partitioning of the node area, and pheromone exploiting on addressing. The algorithm is purely proactive and introduced for large-scale MANETs. Forward and backward ants check the path periodically and reflect the current state of the network. Depending on this path will positively or negatively follow by the ants. Also, pheromone evaporation favors further exploration and removal of out-ofdate paths. This algorithm reserves a path because of hop -byhop routing methodology. The traffic across the network is continually monitored by mobile agent. The demerits of this algorithm are more overload and latency, due probabilistic routing path are not loop free.

Table 1: Summary of ACO based protocols used in this study[10].

\begin{tabular}{|l|l|l|}
\hline Protocol & Path type & Routing type \\
\hline AntNet & Single & Proactive \\
\hline AntHocNet & Single & Hybrid \\
\hline ARA & Multipath & Reactive \\
\hline Ant-AODV & Multipath & Hybrid \\
\hline PERA & Single & Proactive \\
\hline MABR & Single & Proctive \\
\hline
\end{tabular}

\section{CONCLUSION}

Thus we have reviewed some natured algorithm for its different behavior. There is some other algorithms based on natured inspired algorithms for study. Also there is a scope for authors are grateful to the management and

\section{ACKNOWLEDGMENTS}

The authors are grateful to the management and staff of D. J. Sanghavi College of Engineering, Vile Parle, Mumbai, 400056, India, for allowing to use their infrastructure for experimentations.

\section{REFERENCES}

[1] http://en.wikipedia.org/wiki/List_of_ad_hoc_routing_ protocols

[2] T. Srinivasan1, T. NiranjanBabu, V. Mahadevan, M. Nivedita, G. Sahitya, A. Meyyappan, J. V. Sahana: "BFBR A novel Bird Flocking Behavior based Routing for Highly Mobile Ad hoc Networks" In International Conference on Computational Intelligence for Modeling Control and Automation, and International Conference on Intelligent Agents, Web Technologies and Internet Commerce (CIMCA-IAWTIC'06)2006, IEEE

[3] MohitTiwari, ShirshuVarma. "Bird Flight Inspired Clustering based Routing Protocol for Mobile Ad Hoc
Networks". In IJCSNS International Journal of Computer Science and Network Security, VOL.10 No.3, March 2010.

[4] Muddassar Farooq and Gianni A. Di Caro "Routing Protocols for Next Generation Networks Inspired by Collective Behaviors of Insect Societies: An Overview"

[5] G.A. Di Caro, F. Ducatelle, and L.M. Gambardella. AntHocNet: an ant-based hybrid routing algorithm for mobile ad hoc networks." In Proceedings of PPSNVIII, volume 3242 of LNCS, pages 461-470. Springer, 2004. (Best paper award).

[6] G.A. Di Caro, F. Ducatelle, and L.M. Gambardella. "AntHocNet: an adaptive nature-inspired algorithm for routing in mobile ad hoc networks". European Transactions on Telecommunications, 16(5):443-455, 2005.

[7] G.A. Di Caro, F. Ducatelle, and L.M. Gambardella. "Swarm intelligence for Routing in mobile ad hoc networks". In Proceedings of the IEEE Swarm Intelligence Symposium, pages 76-83, Pasadena, USA, June 2005. IEEE Press.

[8] F.Ducatelle,G.A. Di Caro, and L.M. Gambardella."Using ant agents to combine reactive and proactive strategies for routing in mobile ad hoc networks".International Journal of Computational Intelligence and Applications, Special Issue on Nature-Inspired Approaches to Networks and Telecommunications, 5(2):169-184, 2005.

[9] F. Ducatelle, G.A. Di Caro, and L.M. Gambardella. "An evaluation of two swarm intelligence manet routing algorithms in an urban environment". In Proceedings of the 5th IEEE Swarm Intelligence Symposium, St. Louis, USA, September 21-23, 2008.

[10] Vaibhav Godbole, "performance analysis of bio- inspired protocol based on random waypoint mobolity model.

[11] G.A. Di Caro, F. Ducatelle, and L.M. Gambardella. "Studies of routing performance in a city-like test bed for mobile ad hoc networks". Technical Report07-06, IDSIA, Lugano, Switzerland, March 2006.

[12] MesutG unes, UdoSorges, ImedBouazizi "ARA the AntColony Based Routing Algorithm for MANETs" D. J. T. Sumpter. "From bee to society: An agent-based investigation

[13] Mesut Günes, , UdoSorges, ImedBouazizi "ARA the Ant-Colony Based Routing Algorithm for MANETs".

[14] S. Marwaha, C. K. Tham, and D. Srinavasan. "Mobile Agents based Routing Protocol for Mobile Ad hoc Networks". In Proceedings of GLOBECOM, pages 163167, Taipei, Taiwan, November 2002. IEEE Press

[15] J. S. Baras and H. Mehta. "A probabilistic emergent routing algorithm (PERA) for mobile ad hoc networks" In Proceedings of WiOpt, 2003

[16] M. Heissenb üttel and T . Braun. "Ants-based routing in large scale mobile ad-hoc networks". In ITG/GIFachtagung Kommunikation in verteilten Systemen (KiVS 2003), pages 91-99, Leipzig, Germany, 2003. 